\title{
UNA APROXIMACIÓN \\ A LAS RELACIONES ENTRE \\ IDENTIFICACIÓN Y JUSTICIA \\ EN ARGENTINA (1886-1933)
}

MERCEDES GARCÍA FERRARI

Mercedes García Ferrari es Investigadora y Docente

en el Área de Historia del Instituto de Ciencias de

la Universidad Nacional de General Sarmiento.

e-mail: mgarciaferrari@gmail.com

\section{INTRODUCCIÓN}

El objetivo de este trabajo es estudiar la problemática de la identificación individual en la justicia, a partir de la puesta en cuestión de la homogeneidad de las políticas identificatorias de la policía y la justicia a fines del siglo XIX y principios del siglo XX. Se trata de explorar los puntos de contacto, pero muy especialmente las diferencias y la especificidad del proceso de expansión de las prácticas identificatorias de la justicia en la Argentina finisecular.

La hipótesis que se ha sostenido para explicar la distinta aceptación de las tecnologías de identificación en la policía y en la justicia plantea que el honor, valor compartido por amplios sectores sociales en el cambio de siglo, es central para explicar las resistencias judiciales a identificar por medio de tecnologías biométricas a los procesados e incluso condenados. De acuerdo a esta hipótesis, para explorar el proceso de ampliación de las prácticas identificatorias son centrales los significados sociales de las tecnologías, las concepciones acerca del cuerpo y de la legitimidad de su manipulación y registro por parte del Estado. El relato de largo plazo, entonces, apuntaría a explicar este proceso a través de transformaciones culturales: la aceptación de la prácticas identificatorias en la justicia estaría relacionada con la aparición del sistema dactiloscópico, menos invasivo y, a diferencia de la antropometría (asociada a una larga tradición que vinculaba rasgos físicos y condiciones psicológicas y morales) y la fotografía (asociada a su vez a una larga 
tradición de uso del retrato como forma de identificar delincuentes) sin significados estigmatizantes previos ${ }^{1}$.

Este trabajo pretende sumar elementos a esta explicación. Si bien es indudable que los aspectos culturales son muy importantes para explicar las decisiones judiciales, es necesario analizar con mayor detalle la problemática que representó la identificación individual para el sistema judicial. En tal sentido, dos grandes cuestiones - diferentes pero interrelacionadas- pueden ser analizadas: la comprobación de la reincidencia y la constitución de las tecnologías en evidencia forense. La primera surgió como problema a partir de la legislación que establecía la distinta latitud de las penas de acuerdo a la reiteración de las condenas penales de un individuo. Para poder aplicar este tipo de legislación, era necesario determinar la identidad individual y verificar en futuras detenciones si un individuo ya había sufrido condenas anteriores. La constitución de las tecnologías en evidencia forense nos lleva a otra serie de cuestiones relacionadas, por un lado, con la evaluación de la legitimidad científica de los distintos sistemas, y por otro a la delimitación de los alcances de los juicios periciales en el proceso penal. En este trabajo exploraré la primera de las dos instancias, teniendo en el horizonte que en un trabajo posterior ambas deben ser puestas en relación.

Para avanzar en esta indagación, se analizará la legislación penal relativa a la reincidencia entre 1886 y 1921, los códigos de procedimientos en materia penal vigentes para el fuero penal en Capital Federal, territorios nacionales y en la provincia de Buenos Aires, y dos proyectos de ley de creación de archivos nacionales de reincidencia: el proyecto de Ernesto Quesada, Comprobación de reincidencias (1901), y el de Emilio Gouchon para la creación de una Oficina Nacional de Identificación y estadistica en materia penal (1904). Si bien en estos años hubo varios proyectos de creación de archivos de identificación no policiales, estos dos son los únicos que se centraron en la identificación criminal y plantearon la necesidad de crear archivos a nivel nacional dependientes del Ministerio de Justicia e Instrucción Pública.

\footnotetext{
${ }^{1}$ Ver Kristin Ruggiero, Modernity in the Flesh. Medicine, Law and Society in Turn-of-the-Century Argentina, Stanford, Stanford University Press, 2004; Mercedes García Ferrari, «Una marca peor que el fuego. Los cocheros de la ciudad de Buenos Aires y la resistencia al retrato de identificación», en: Lila Caimari, (comp.), La ley de los profanos. Delito, justicia y cultura en Buenos Aires (1870-1940), Buenos Aires, Fondo de Cultura Económica, 2007.
} 
Esta indagación propone también reposicionar el problema en una serie de cuestiones relacionadas con las capacidades burocráticas del Estado y la homogeneización del sistema penal a nivel nacional. Por otro lado, este análisis ayuda a pensar las diferencias entre las prácticas identificatorias en la policía y la justicia, y muestra que el problema de la legitimidad científica de los sistemas de identificación tuvo un peso sumamente diferente en ambas instituciones.

\section{LA REINCIDENCIA EN EL CÓDIGO PENAL}

En este apartado propongo dejar de lado la mirada de la problemática policial y urbana, explorada en otros trabajos ${ }^{2}$ para aproximarme al marco legislativo relativo a la reincidencia a fines del siglo XIX y principios del XX. La creciente importancia de la reincidencia como factor de modulación de las penas en este período es bien conocida. Si bien el problema de la «delincuencia habitual» formó parte de preocupaciones extendidas socialmente y de ningún modo exclusivas de la justicia, y el impulso para la búsqueda de sistemas eficaces y económicos de identificación se puede rastrear sin duda en transformaciones más amplias de la sociedad y el Estado, la existencia de códigos que penaban de forma diferenciada a los reincidentes justificó la apertura de oficinas de identificación en los departamentos de policía argentinos. La historia de la identificación en este período estuvo estrechamente vinculada con la búsqueda de sistemas que posibilitaran la comprobación de la reincidencia.

Hasta la creación del Registro Nacional de Reincidencia y Estadística Criminal y Carcelaria, dependiente del Ministerio de Justicia e Instrucción Pública (1933) los jueces se basaron en la información suministrada por las oficinas policiales para establecer la latitud de las penas. El Código Penal de 1886 establecía que las penas serían agravadas de «haber sido el culpable castigado anteriormente por delito a que la ley señale igual o menor pena» (Art. 84, inc. 19) o si fuera «reincidente de delitos de la misma especie» (Art. 84, inc. 20) ${ }^{4}$. Evidentemente, esto sólo podía

2 Mercedes García Ferrari, Ladrones conocidos/sospechosos reservados. Identificación policial en Buenos Aires, 1880-1905, Buenos Aires, Prometeo, 2010.

${ }^{3}$ Ley 11.752, Registro Nacional de Reincidencia y Estadística Criminal y Carcelaria, Boletín Oficial, 13/10/1933.

${ }^{4}$ Ley 1.920, Código Penal, sancionada el 25/11/1886, promulgada el 07/12/1886. En este trabajo no se hará referencia a los debates en torno a la reincidencia sino únicamente a su comprobación por medio de tecnologías de identificación. 
cumplirse si era posible establecer la identidad de los procesados. A su vez implicaba un importantísimo esfuerzo de transformación burocrática: unir el cuerpo del sujeto al archivo, disponer de un archivo judicial exhaustivo y lograr un vínculo eficiente entre el archivo judicial y los registros de identidad. Por otro lado, a diferencia del Código de Tejedor que establecía un plazo máximo de diez años desde la última condena para el agravamiento de las penas, el nuevo Código Penal no ponía límites temporales, multiplicando así el problema del archivo judicial.

Pasarían más de cuarenta ańos para que la comprobación de la reincidencia pudiera hacerse efectiva a nivel nacional. Si en la década de 1890 era todavía azarosa la identificación de quien hubiera sido condenado más de una vez en la Capital, era prácticamente imposible identificar a quien reincidiera en distintas jurisdicciones. En el contexto del Estado argentino en formación, encontrar un método de identificación eficaz fue mucho más sencillo que lograr la construcción de un registro de antecedentes unificado a nivel nacional.

De este modo, además de las aspiraciones policiales de construir un conocimiento pormenorizado acerca de los sujetos sobre quienes concentraba la vigilancia, la mejora en los sistemas de identificación y la mayor fluidez en la información de antecedentes judiciales estaba también entre las prioridades del poder judicial. En 1886, un año antes de que se iniciaran la gestiones para la apertura de la Oficina Antropométrica, una Orden del Día de la Jefatura de Policía de la Capital intentaba «uniformar el procedimiento» de remisión de presos para «evitar, en lo posible, la aglomeración de nombres». Nombres que se acumulaban asociados en el mejor de los casos a una fotografía, en otros únicamente a una vaga descripción física. No sólo faltaba un sistema de identificación, tampoco se mantenía la mínima uniformidad en las filiaciones lo que tornaba «ilusorias las disposiciones del Código Penal sobre las reincidencias» ${ }^{5}$. En 1887 se comenzó a planificar la creación de una oficina de identificación antropométrica en la Policía de la Capital y dos años más tarde, en 1889, se inauguró.

${ }^{5}$ Orden del Día, 19/08/1886. Esta disposición establecía una modificación en el libro de «Entradas de Presos»: se agregarían casilleros para poder completar en cada caso el «verdadero nombre de los detenidos, especialmente cuando se tratase de crímenes o delitos» (es decir, en aquellos casos en los que se podía aplicar un agravamiento de pena), «nacionalidad, edad, estado, profesión, color, si lee y escribe y su tiempo de residencia en el país», además de otros nombres y apodos. 
Si para la policía establecer la identidad individual formaba parte de un conjunto amplio de preocupaciones fuertemente vinculadas al control de un territorio, para los jueces era fundamental, para poder dictar sentencia, confiar en la exactitud de la información sobre los sujetos a quienes impondrían una pena.

En 1895 se reforzó la importancia de la reincidencia para la determinación de las penas: quienes reincidieran por segunda vez en Capital y territorios nacionales debían cumplir su pena en los territorios nacionales del sur'. En 1903 se estableció un límite temporal de diez años para la reincidencia por delitos de la misma especie $^{7}$. Finalmente en 1921 el nuevo Código Penal estableció que «habrá reincidencia siempre que el condenado por sentencia firme a una pena privativa de libertad, dictada por cualquier tribunal del país, cometiere nuevo delito, aunque hubiese mediado indulto o conmutación ${ }^{8}$. Mantuvo la reclusión en un "paraje de los territorios del Sud» para los reincidentes por segunda vez con penas mayores a dos ańos y agregó la reclusión en el sur por tiempo indeterminado en una serie de casos: dos condenas a reclusión y una a prisión por más de tres años; tres condenas a prisión por más de tres años o una de reclusión y dos de prisión de tres años o menos; cuatro condenas a prisión, si una de ellas era mayor a tres años; cinco condenas a prisión de tres años o menores. También se tenían en cuenta las condenas en el exterior para delitos que pudieran dar lugar a extradición según la ley argentina y no se tomarían en cuenta las penas que hubiera sufrido el condenado antes de cumplir los 21 años.

En 1921 los archivos de identificación estaban sin duda muy desarrollados en los departamentos de policía, encargados además del intercambio internacional de antecedentes e información de identidad. Sin embargo, y a pesar de la presentación de diversos proyectos de ley, no se había creado un registro que centralizara la información a nivel nacional, como tampoco se había logrado crear un archivo dependiente directamente del Ministerio de Justicia e Instrucción Pública.

En el período estudiado, la justicia necesitó del archivo policial para establecer en cada caso la latitud de las penas. Esto fue motivo de constantes tensiones.

${ }^{6}$ Ley 3335, Reincidentes: cumplimiento de penas en territorios del Sur. Ver: Lila Caimari, Apenas un delincuente. Crimen, castigo y cultura en la Argentina, 1880-1955, Buenos Aires, Siglo XXI, 2004, pp. 63-73.

7 Ley 4189, Código Penal, modificaciones de la Ley 1920, Art. 15.

${ }^{8}$ Ley 11179, Código Penal, promulgado el 29 de octubre de 1921. Título VIII, Reincidencia, Arts. 50-53. 
En 1889 se abrió la Oficina de Identificación Antropométrica de la Policía de la Capital porque «no hay actualmente base fija para la constatación de la identidad (...) de donde resulta que la circunstancia agravante de la reincidencia no puede en la mayor parte de los casos ser establecida con precisión»". Sin embargo, los objetivos de la justicia y la policía pronto se diferenciaron: para la justicia sólo era necesario identificar a quienes hubieran sido condenados, mientras que la policía buscaba ampliar el espectro de sujetos identificados. Por otro lado, tampoco era equivalente la preocupación por la exactitud del sistema empleado. La toma de las diez huellas digitales se implementó desde 1891 en la policía de la Provincia de Buenos Aires, cuando no había ninguna certeza acerca de su efectividad. En 1905 el sistema dactiloscópico fue adoptado también por la policía de la Capital, cuando había recibido muchos apoyos internacionales pero la discusión científica no estaba aún absolutamente zanjada. En los departamentos de policía la identificación pronto fue considerada una práctica "preventiva», y se expresó el objetivo de construir, si fuera posible, un archivo de la totalidad de la población. Para la policía era fundamental encontrar un sistema fácilmente aplicable a grandes poblaciones con los recursos institucionales disponibles, aunque pudiera arrojar algún margen de error. Por lo tanto, el derrotero de la implementación de distintas tecnologías de identificación en los departamentos de policía no necesariamente nos acerca a conocer su grado de aceptación en el sistema judicial ${ }^{10}$.

\section{LOS CÓDIGOS DE PROCEDIMIENTOS EN MATERIA PENAL}

El Código Penal de 1886, de alcance nacional, establecía entonces que era necesario tener en cuenta la reincidencia para determinar la latitud de las penas y, a partir de 1895, también dónde debían cumplirse las mismas. La cuestión relativa

\footnotetext{
9 Orden del Día, 03/04/1889, Policía de la Capital.

${ }^{10}$ Aunque, por distintas causas que analizaremos más adelante, todo indica que el agravante de la reincidencia fue muy poco considerado en este período, es difícil avanzar en este punto en tanto no se haga un trabajo de investigación sobre los fallos judiciales. No sabemos hasta qué punto los jueces tomaron en cuenta la información que recibían de las Oficinas de Identificación para alargar las penas o decidir, por ejemplo, el envío de un condenado al penal de Ushuaia, tampoco si esta respuesta fue homogénea. Por otro lado, sabemos gracias a los trabajos de Kristin Ruggiero, que en algunos casos hubo jueces que dieron lugar a los pedidos de exención de la identificación o a la destrucción de registros, pero no es posible evaluar el verdadero alcance de estas resistencias judiciales a los nuevos métodos.
} 
a la forma en que debía comprobarse la reincidencia se estipulaba, sin embargo, en los códigos de procedimientos, de alcance local.

El Código de Procedimientos en lo Criminal, vigente para el fuero federal y los tribunales ordinarios de la Capital y territorios nacionales, promulgado en 1888, establecía que «a fin de que puedan servir como prueba de identidad, se harán constatar con la minuciosidad posible las señas del procesado» (Art. 271) $\mathrm{y}$ «si el presunto reo, al recibirle su declaración, negare su nombre y apellido, su nacionalidad o domicilio, o lo fingiese, se procederá a identificar su persona por medio de testigos de reconocimiento, y, en su defecto, por los medios que parezcan oportunos» (Art. 270) ${ }^{11}$. En 1896, la provincia de Buenos Aires promulgó con algunas adaptaciones el código federal ${ }^{12}$.

Por lo tanto, luego de haberse abierto la Oficina de Identificación Antropométrica en la Capital y cuando en la provincia de Buenos Aires ya se había avanzado en el perfeccionamiento del sistema dactiloscópico ${ }^{13}$, se promulgaron códigos de procedimientos que no hacían ninguna mención a los nuevos métodos de identificación o sus oficinas. Ernesto Quesada diría que «la justicia argentina libra a los mismos procesados el comunicar si son o no reincidentes» ${ }^{14}$. La palabra del procesado o la de testigos eran las principales fuentes para establecer la identidad, además de las filiaciones que pudiera elaborar la policía ${ }^{15}$. De todos modos, estos códigos dejaban abierta la resolución del problema: se podían aplicar, además de los métodos tradicionales, los «medios que parezcan oportunos», por lo tanto

\footnotetext{
${ }^{11}$ Ley № 2372, Código de Procedimientos en Materia Penal.

${ }^{12}$ Código de Procedimientos en lo Criminal para la provincia de Buenos Aires sancionado por Ley de 11 de Junio de 1896, Edición Oficial. Los equivalentes a los artículos 270 y 271 del Código de Procedimientos de 1888, eran los artículos 217 y 218 del Código de la provincia de Buenos Aires (con el mismo texto).

${ }^{13}$ En 1891, al abrirse la Oficina de Identificación de la policía de la Capital de la provincia de Buenos Aires se realizó la toma de las 10 huellas digitales de 23 detenidos mediante una clasificación basada en los trabajos de Francis Galton. En los años siguientes, Juan Vucetich continuó implementando el sistema y modificando la forma de clasificar las impresiones digitales hasta llegar a su sistema de cuatro tipos fundamentales. En 1896, la Oficina de Identificación de la Policía de la provincia de Buenos Aires utilizaba el sistema dactiloscópico, el sistema de filiación «Provincia de Buenos Aires» (también desarrollado por Vucetich) en reemplazo del bertillonage y la fotografía de identificación. ${ }^{14}$ Ernesto Quesada, Comprobación de la reincidencia, Buenos Aires, Imprenta y Casa Editora de Coni Hermanos, 1901, p. 58.

${ }^{15}$ Como hemos visto, estas filiaciones no proporcionaban ninguna certeza al juez, por lo que en la práctica salvo que el procesado fuera «conocido» por la policía o la alcaldía no se establecía la reincidencia.
} 
era posible acudir a las oficinas de identificación pero no resultaba obligatorio ${ }^{16}$. Por otro lado, las diferencias locales (tanto de una provincia a otra, como de un tribunal o incluso de un juez en particular a otro) provocaban grandes diferencias en la aplicación de la legislación penal. Como veremos más adelante, por esta razón Quesada planteaba que la comprobación de la reincidencia no podía formar parte del código de procedimientos sino que debía responder a una ley nacional complementaria del código penal. La unidad territorial de aplicación de la legislación penal, sólo podía respetarse si se homogeneizaba el procedimiento a través del cual se determinaba la identidad de los procesados y se lo vinculaba con sus antecedentes penales. El código de procedimientos de 1888, con algunas modificaciones, continuó vigente en el fuero federal y para Capital y territorios nacionales durante todo el período estudiado.

En 1906, se promulgó para la provincia de Buenos Aires un código procesal sumamente innovador en el contexto internacional ${ }^{17}$. En el título dedicado a la «Identidad del presunto reo» se mantuvo el reconocimiento por testigos o «los medios que parezcan oportunos» (Art. 432), pero se agregó un nuevo artículo que planteaba:

«A fin de que pueda servir como prueba de identidad, la Oficina de Identificación tomará la individual dactiloscópica del procesado, sin perjuicio de las demás señales personales del mismo que, por otros medios, hiciera constar la autoridad respectiva» (Art. 433) ${ }^{18}$.

Por un lado establecía qué sistema de identificación debía ser considerado como prueba de identidad, y por otro que la Oficina de Identificación, dependiente de la Policía de la Provincia de Buenos Aires, era la responsable de este procedimiento. También el nuevo código especificaba cómo proceder cuando no se pudiera acreditar la edad del procesado: por medio de la inscripción del nacimiento en el

\footnotetext{
${ }^{16}$ Volveré más adelante sobre el tema pero la dependencia responsable de brindar informes a la justicia en la Capital fue la alcaldía.

${ }^{17}$ En principio, este fue el primer código de procedimientos que incorporó la dactiloscopia como forma de comprobar la identidad del delincuente.

${ }^{18}$ Código de Procedimientos en materia penal, Edición Oficial, La Plata, Taller de Impresiones Oficiales, 1906. Este código también fue innovador en la consideración de las huellas digitales como prueba forense: se estableció su incorporación en las inspecciones oculares; la intervención de la Oficina de Identificación en todos los casos en los que hubiera evidencia dactiloscópica y la toma de la individual dactiloscópica de cadáveres involucrados en un proceso penal antes de ser inhumados.
} 
Registro Civil o partida de bautismo (Art. 436). Este procedimiento reemplazaba, cuando fuera posible, la estimación por medio de un examen físico de los médicos de tribunales ${ }^{19}$. Por último, la Oficina de Identificación era la encargada de brindar los antecedentes judiciales de los procesados que debían ser enviados al juez junto con la individual dactiloscópica dentro de las 48 horas de realizada la identificación.

La sanción de este código de procedimientos estuvo relacionada con la creciente importancia de Juan Vucetich en la Policía de la provincia de Buenos Aires, en un momento en que comenzó a recibir fuertes apoyos internacionales y el sistema dactiloscópico se difundió en varios países latinoamericanos. Un año antes, en 1905, la Policía de la Capital había adoptado el sistema y se había establecido el canje de individuales dactiloscópicas entre varios países del Cono Sur (Argentina -Capital y Provincia de Buenos Aires-, Brasil, Chile, Uruguay). La Oficina de Identificación dirigida por Vucetich en la ciudad de La Plata era un referente mundial en materia de identificación por medio de huellas digitales y la que mayor experiencia tenía en la implementación práctica del sistema. El código de procedimientos era sin duda parte del posicionamiento de la provincia de Buenos Aires en la frontera de la ciencia ${ }^{20}$.

${ }^{19}$ Si bien la preocupación central para los contemporáneos era la comprobación de la reincidencia, hay que tener en cuenta también el problema de la determinación exacta de la edad de los procesados. Por ejemplo, la pena de muerte no se imponía a menores de edad y hombres mayores de 70 años; los menores de edad y los mayores de 60 años no cumplirían pena de presidio sino de penitenciaría; los menores de 10 años en todos los casos y los mayores de 10 y menores de 15 que no hubieran actuado con discernimiento estaban exentos de pena (Art. 59, Art. 62; Art. 81, Ley 1920 Código Penal). Una diferencia de un año podía determinar la vida o la muerte, la exención de pena o la condena, en un momento en el que era frecuente desconocer la propia edad exacta y no tener documentos probatorios de la misma. ${ }^{20} \mathrm{Si}$ bien en este trabajo no se estudia el uso forense de las huellas digitales, es muy probable que la Oficina de Identificación realizara peritajes dactiloscópicos desde la década de 1890 como parte de un procedimiento no escrito pero bastante regular. En 1892, se consideraron por primera vez las huellas digitales como parte de una investigación policial en el doble homicidio de los hijos de Francisca Rojas, que tuvo lugar en Necochea. En este caso el pesquisa Eduardo Álvarez, enviado desde la capital provincial para supervisar la investigación, encontró en la escena del crimen huellas digitales en sangre y llevó esta evidencia junto con las individuales dactiloscópicas de los dos sospechosos a la Oficina de Identificación. El sumario estuvo a cargo del comisario local, por lo que incluso si se encontrara el expediente judicial, es posible que no haya referencias al uso pericial de las huellas digitales. El caso se resolvió por medio de la confesión de la madre de los niños, al ser confrontada con la evidencia de que sus huellas coincidían con las encontradas en la escena del crimen (Legajo № 84: El crimen de Necochea, Eduardo M. Álvarez, Informe al Jefe de Policía Guillermo Nunes, 12 de julio de 1892, Colección Vucetich). En 1899, la sentencia de Audifracio González por homicidio muestra que el juez 
En 1915, la provincia de Buenos Aires sancionó un nuevo código de procedimiento penal ${ }^{21}$ que ratificó la incumbencia de la Oficina de Identificación para comprobar la reincidencia. Los comisarios o jueces estaban a cargo de tomar las huellas digitales de los detenidos y enviarlas a la Oficina que debía responder en 48 horas con los antecedentes y la ficha dactiloscópica (Art. 206 y 207).

\section{EL PROYECTO QUESADA}

En 1901, Ernesto Quesada elevó un informe al Ministro de Justicia e Instrucción Pública Osvaldo Magnasco que debía servir como base para un proyecto de ley del Poder Ejecutivo que reglamentara la comprobación de la reincidencia ${ }^{22}$. Se trata de un extenso estudio que explora distintas legislaciones sobre reincidencia y las formas de comprobarla en una perspectiva comparativa internacional. Es, sin duda, el trabajo más importante sobre el tema escrito en el período, en el que se reúnen las principales discusiones sobre el tema, la legislación internacional, la situación en Argentina tanto a nivel legislativo como en la práctica en la Alcaldía y la Oficina Antropométrica de la Capital, y una discusión sobre los distintos sistemas de identificación existentes ${ }^{23}$.

\footnotetext{
encargó a la Oficina de Identificación las pericias dactiloscópicas y químicas, que fueron remitidas a la oficina correspondiente (Causa № 1370, Sentencia de Audifracio González, La Plata, Mayo de 1899 -copia-Colección Vucetich). La aceptación de la huella digital como evidencia forense es sin duda un tema a explorar. Tanto en los trabajos de Vucetich, como en los de su discípulo el Dr. Luis Reyna Almandos y en la documentación del Fondo Particular de Juan Vucetich, este es un área llamativamente relegada, aunque algunas evidencias llevan a pensar que la Oficina de Identificación era consultada regularmente para realizar peritajes. Por el momento, la hipótesis para explicar este vacío en el archivo es que Vucetich buscó (y logró) el título de Perito Identificador tanto de la provincia de Buenos Aires (1909) como de la Nación (1910), justamente para separar el conocimiento experto de la institución policial, tratando de posicionar el nuevo saber dentro del campo científico. La evidencia reunida hasta el momento indica que el pedido de peritajes se dirigía a la Oficina de Identificación y no a Vucetich en forma personal. Por otro lado, Vucetich no fue especialmente innovador en la investigación científica del delito.

${ }^{21}$ Código de Procedimiento Penal, sancionado en enero 13 de 1915, Edición Oficial, La Plata, Talleres de Impresiones Oficiales, 1915.

${ }^{22}$ El proyecto de ley no fue presentado por el Poder Ejecutivo pero se publicó el informe en 1901: Ernesto Quesada, Comprobación de la reincidencia. Proyecto de Ley presentado al señor Ministro de Justicia e Instrucción Pública Doctor Don Osvaldo Magnasco, Buenos Aires, Imprenta y Casa Editora de Coni Hermanos, 1901.

${ }^{23}$ Quesada intervino fuertemente en la discusión sobre la validez de la antropometría y la dactiloscopia.
} 
El proyecto de ley propuesto por Quesada es el primero en Argentina que intentó reglamentar la comprobación de la reincidencia y, según el autor, es también el primero en el mundo ${ }^{24}$. Inauguró una forma de entender la identificación judicial que finalmente se concretaría en el primer Registro Nacional de Reincidencia y Estadística Criminal (1933). El proyecto planteaba varias cuestiones: la importancia de unificar el procedimiento de identificación en todo el territorio y de crear una oficina a nivel nacional, así como la necesidad de separar la identificación judicial de la policial o civil.

Durante las primeras décadas del siglo XX se estaba discutiendo quiénes serían los encargados de la construcción de archivos, y muchos conflictos pueden ser leídos a la luz de las divergentes posiciones al respecto. La policía pretendía ocuparse de la identificación en general: proveer datos a la justicia desde sus oficinas de identificación; encargarse de la identificación civil por medio de la expedición de cédulas de identidad, pasaportes, cartas de ciudadanía, carnets o libretas para determinados gremios, certificados de antecedentes; registrar a quienes fueran detenidos por contravenciones o averiguación de identidad ${ }^{25}$. Otra posición, sostenida

Para la redacción de su informe entró en contacto con Vucetich y realizó visitas a su oficina de La Plata, iniciando una estrecha relación. Las visitas al gabinete de Vucetich convencieron a Quesada de la superioridad de la dactiloscopia (cambiando su previo apoyo a la antropometría). Vucetich se encargó personalmente de difundir el libro de Quesada y le proporcionó su lista de contactos internacionales relacionados con la identificación para que enviara su trabajo. Sin embargo, años más tarde (1909) se desató una fuerte polémica entre Quesada por un lado y Vucetich y su discípulo Reyna Almandos por otro, a raíz del nombramiento de Vucetich como primer perito dactilóscopo de la provincia de Buenos Aires y al año siguiente como primer perito dactilóscopo de la Nación. En este período se estaba reglamentando tanto la formación de los peritos e idóneos como los alcances de los peritajes. Traductores, intérpretes, calígrafos y por último dactilóscopos constituían áreas de expertise que se estaban definiendo en estos años. En 1883, el primer perito calígrafo juró ante la Corte Suprema de la Nación (sin embargo, recién en 1973 se reglamentó por ley -20.243- la profesión para la Capital Federal), en 1907 se estableció la forma de obtener el título de Perito traductor e intérprete en la provincia de Buenos Aires (Arturo Acosta Álvarez, Sobre traducciones Judiciales, La Plata, s/d, 1908) y en 1908 Vucetich pidió ser nombrado Perito Dactilóscopo de la provincia de Buenos Aires, título que obtuvo a nivel provincial en 1909 y nacional en 1910 (fue encargado, además, de crear un programa de formación y un examen para futuros nombramientos, y de designar de manera transitoria a quienes podían oficiar de idóneos). ${ }^{24}$ Hace falta profundizar el estudio comparativo para corroborar esta afirmación, especialmente tratándose de proyectos de ley y no de leyes efectivamente sancionadas. En 1901 se presentó también un proyecto de Registro de Vecindad y Cédula de Vecindad en la provincia de Buenos Aires.

${ }^{25}$ Esta fue la opción que se impuso de hecho a principios del siglo XX a través del crecimiento de las funciones de las oficinas de identificación policiales. En 1908 el Jefe de la Oficina de Identificación de la Policía de 
por Vucetich, buscaba crear una oficina independiente tanto de la policía como de la justicia, encargada de todos los temas relativos a la identidad de las personas que se dedicaran, además, a la investigación científica ${ }^{26}$. Por último, los proyectos que analizaremos en este trabajo apuntaban a deslindar las necesidades judiciales de comprobación de la reincidencia de otras instituciones, especialmente de la policía. Son proyectos que no apuntaban a dar una respuesta integral al problema de la creación de archivos de identificación, sino exclusivamente a solucionar el problema judicial de comprobación de la reincidencia mediante la creación de una Oficina Nacional dependiente del Ministerio de Justicia e Instrucción Pública (a diferencia de otros proyectos cuyas oficinas dependían del Ministerio del Interior o de Gobierno en la provincia de Buenos Aires) ${ }^{27}$.

La discusión sobre la reincidencia incluye un abanico de cuestiones de las que aquí sólo abordaremos la relacionada con su comprobación. De todos modos, es necesario decir que la preocupación central de Quesada al escribir su proyecto estaba centrada en la reincidencia como un "gravísimo estado patológico de la sociedad» y lograr un método efectivo para comprobarla era para él la única forma de «extirpación de un verdadero cáncer social ${ }^{28}$. Aquí, como ya he dicho en la introducción, me limitaré a buscar algunas claves para profundizar en el problema de identificación desde el punto de vista judicial y ampliar la explicación de

la Capital presentó un proyecto al Ministerio del Interior en el que proponía crear una Oficina Nacional de Identificación sobre la base de la oficina de la policía porteña. Este proyecto incluía el registro cívico para fines electorales (que nunca estuvo a cargo de la policía) y la fiscalización policial de las elecciones en materia de identidad de los votantes. Nicéforo Castellano, Oficina Nacional de Identidad. Proyecto presentado al Ministro del Interior, 6 de Abril de 1908, Buenos Aires, Escuela Tipográfica del Colegio Pío IX, 1908.

${ }^{26}$ En esta línea se presentaron dos proyectos de carácter provincial en la legislatura de la provincia de Buenos Aires. El proyecto de Octavio R. Amadeo que se discutió en la cámara de diputados de la provincia en 1910 y el proyecto de Vucetich que se transformó en Ley de Registro General de Identificación, promulgada el 20 de julio de 1916 y derogada un año más tarde con la intervención nacional a la provincia de Buenos Aires. En estos proyectos era central desligar a la policía de la identificación, tanto criminal como civil, y dar un carácter de oficina científica al nuevo organismo. La estructura a nivel provincial se apoyaba fuertemente en las oficinas locales de Registro Civil y no en las comisarías (de todos modos, en 1904 Vucetich apoyó el proyecto de Gouchon).

${ }^{27}$ Del mismo modo que los proyectos de Quesada y Gouchon se referían con exclusividad a la identificación criminal, hubo proyectos dedicados exclusivamente a la identificación civil: el Proyecto de Ley de Registro de Vecindad para la provincia de Buenos Aires, presentado por Bernardo de Irigoyen y Tomás R. García en 1901 y el proyecto de Formación de un Registro de Vecindad presentado por Nicolás Avellaneda en 1909. ${ }^{28}$ Ernesto Quesada, op. cit., pp. 9-10. 
las «resistencias» judiciales a utilizar tanto las oficinas de identificación policiales como a aceptar las nuevas tecnologías de identificación.

El primer gran problema que encontraba Quesada en la legislación vigente sobre identificación era la imposibilidad de homogeneizar las prácticas identificatorias en todo el territorio nacional:

«Dado nuestro régimen federal de gobierno, las leyes de fondo son de atribución nacional, pero las de forma corresponden a las provincias. De ahí la primera dificultad, porque las leyes procesales no son uniformes en todo el país. Cada organización judicial local observa, en lo relativo a la filiación de los presos, prácticas arbitrarias y rutineras ${ }^{29}$.

Es decir, la primera cuestión que se planteaba era que la forma de comprobar la reincidencia resultaba indisociable de su consideración para la determinación de la pena. Pero estaban establecidas en códigos diferentes: el Código Penal que regía en todo el territorio nacional y exigía un trato idéntico de todos los habitantes ante la ley penal, y una diversidad de códigos de procedimientos que en la práctica establecían una fuerte desigualdad. Este era un punto muy difícil de resolver ya que los códigos de procedimientos eran claramente potestad de los estados provinciales y los intentos de reglamentar de manera general cuál debía ser el sistema de identificación utilizado en toda la república chocaba con este requisito constitucional. Quesada era conciente de que «podría objetarse a esta ley que invade las autonomías provinciales, por cuanto reglamenta un trámite de procedimiento». Buscó, entonces, una solución al problema planteando que la Ley de Comprobación de la Reincidencia no formaría parte del Código de Procedimientos sino que sería un anexo del Código Penal porque, argumentaba, «no se trata de una ley de forma, sino de fondo, no se extralimita interviniendo en la substanciación de los procesos, sino que exige la comprobación de la reincidencia, que es una disposición del Código Penal $»^{30}$. No sabemos qué aceptación pudo tener este argumento, pero el tema aparece claramente en las discusiones del proyecto Gouchon que veremos en el próximo apartado, llevando a un callejón sin salida.

Una segunda cuestión que preocupaba a Quesada era que los códigos de procedimientos, tanto en el fuero federal, de Capital y territorios como de la provincia

29 Ídem, p. 55.

30 Ídem, p. 167. 
de Buenos Aires (hasta 1906), estipulaban un procedimiento basado en la declaración del procesado o de testigos (si se negaba a dar información personal) y la "constancia minuciosa» de las «señas» del procesado ${ }^{31}$. Nada obligaba al juez a pedir informes a las oficinas de identificación. La Oficina Antropométrica de la Policía de la Capital había pedido en 1889 a la Cámara de Apelaciones que adoptara una medida general y obligatoria, ya que en la orden del día que reglamentó su apertura se especificaba que debía pedir caso por caso autorización a los jueces para llevar adelante la rutina del bertillonage (y esto raramente sucedía). La policía fracasó en su pedido y se estableció que «la indicada medida importaría un vejamen a los procesados». Este no era el único problema. Para que la comprobación de la reincidencia fuera equivalente para todos los imputados era necesario que los archivos fueran exhaustivos. Al igual que en el caso de la unidad territorial, un registro aleatorio de procesados terminaba en una aplicación despareja del Código Penal.

Además de la identificación parcial, Quesada consideraba que la antropometría tenía problemas propios como sistema de identificación que complicaban su aceptación en la justicia. Más allá de la oficina de Bertillon, en ningún lugar se logró la sistematicidad necesaria para garantizar resultados fiables. Varias mediciones de un mismo sujeto arrojaban otros tantos resultados diferentes. Si bien existía una tabla de tolerancia para las distintas medidas, estos parámetros eran habitualmente sobrepasados en las mediciones locales. Por otro lado, la antropometría no era un método positivo de identificación sino que, a través de una serie de medidas, lograba una aproximación probabilística a la identidad. Por lo tanto, si el juez decidía pedir informes sobre la identidad de un procesado a la Oficina Antropométrica y ésta respondía que se trataba de un reincidente, el sujeto podía mantenerse firme en la negativa de ser la misma persona que figuraba en el archivo y el juez, dado el carácter probabilístico del sistema, no tenía argumentos para considerar la reincidencia. Como la institución responsable de la identificación era la Alcaldía, y la Oficina Antropométrica constituía sólo un auxiliar de la misma, los jueces generalmente solicitaban los informes de antecedentes directamente a la Alcaldía que continuaba tomando las filiaciones tradicionales ${ }^{32}$.

${ }^{31}$ Art. 270 y 271 del Código de Procedimientos, op. cit., 1888 y Art. 217 y 218 del Código de Procedimientos, op. cit., 1896.

${ }^{32}$ Como ya he dicho, hace falta un trabajo de investigación que permita saber cuál fue la influencia real de la legislación sobre reincidencia en la latitud de las penas. 
En tercer lugar, Quesada diferencia claramente en su trabajo los objetivos policiales de los judiciales para construir archivos biométricos:

«La policía tiene funciones de otro orden y ciertamente un personal experto en su servicio de investigaciones, y que conoce directamente a casi todos los delincuentes temibles de esta capital. Su atención se encuentra absorbida por esa lucha diaria y sin cuartel; y no puede exigírsele que se preocupe de perfeccionar resortes auxiliares de la justicia, como es en todo país el servicio de identificación (...) Para sus necesidades internas tienen las policías sus reglas y procedimientos, a las veces verbales (...) pero la justicia, que nada tiene que ver con eso, necesita otra cosa ${ }^{33}$.

A diferencia de lo que pretenderá Vucetich (quitar a la policía -y en especial a la comisaría de investigaciones- toda intervención en la identificación humana, ya fuera civil o criminal), para Quesada los procedimientos policiales (desprolijos, orales, sin necesidad de reglamentación clara, orientados por la «lucha diaria y sin cuartel» contra el delito) no debían ser modificados. Pero la información que sirviera a los jueces para dictar sentencia no podía provenir de «informes meramente policiales». Como contracara de la identificación policial, la judicial debía ser exhaustiva, reglamentada, regida por claros procedimientos escritos, homogénea en todo el territorio nacional, orientada únicamente a la comprobación de la reincidencia y debía utilizar sistemas que fueran científicamente superiores. Por el contrario, los archivos policiales debían organizarse de acuerdo a un saber eminentemente local: no eran equivalentes las necesidades de una gran ciudad que las de un pequeño pueblo donde los agentes conocían a los delincuentes uno a uno. De acuerdo al proyecto Quesada, los jueces recibirían las filiaciones de los procesados «en uso en cada lugar» pero agregarían una individual dactiloscópica tomada en sede judicial. Una vez dictada la sentencia, sería enviada al archivo nacional junto con la individual dactiloscópica y la filiación policial en cualquier sistema. Una vez dictada la sentencia, esta última sería enviada al archivo nacional junto a la filiación policial. Cuando los jueces solicitaran informes para comprobar la reincidencia, tomarían las huellas digitales y las enviarán al archivo. De esta manera, en tanto se homogeneizaran los procedimientos, Quesada intentaba una solución de compromiso entre el Código Penal nacional y los códigos de procedimientos provinciales, ya que se

\footnotetext{
${ }^{33}$ Ernesto Quesada, op. cit., pp. 143-144.
} 
mantenían las formas de filiación locales y se agregaba una individual dactiloscópica independiente establecida por una ley anexa al Código Penal.

El informe de Quesada al Ministro de Justicia no se concretó en un proyecto de ley efectivamente presentado al Congreso. Sin embargo, muchas de sus ideas fueron retomadas en el proyecto Gouchon que, como veremos, fue discutido y aprobado en la Cámara de Diputados de la Nación en 1905.

\section{EL PROYECTO GOUCHON}

El 11 de julio de 1904, Emilio Gouchon presentó en la Cámara de Diputados de la Nación un proyecto de ley de Oficina Nacional de Identificación y Estadistica en Materia Penal. Con algunas modificaciones, la Comisión de Códigos integrada por Argerich, Luque, Aldao y Carreño, lo sometió a discusión en la sesión del 12 de julio de 1905.

En muchos aspectos, el proyecto seguía los lineamientos del escrito por Quesada en 1901, pero agregaba fundamentalmente dos novedades: la importancia de generar información estadística a nivel nacional que pudiera servir para la «defensa social» $\mathrm{y}$ la relación de esta oficina con otras del exterior. Argerich presentó el proyecto como respuesta a «una de las mayores preocupaciones del gobierno [que] es la defensa contra el delincuente» ${ }^{34}$. Por otro lado, la dactiloscopia era protagonista en este proyecto para cuya redacción la comisión había recibido informes de Vucetich.

Me interesa especialmente recorrer cuatro cuestiones que se suscitaron en el debate legislativo y arrojan luz sobre algunos problemas centrales para pensar la relación entre identificación y justicia en este período.

En primer lugar, apenas abierto el debate se planteó el problema de la dependencia institucional de la oficina: ¿por qué abrir una nueva oficina si la policía de la Capital, que ya disponía de una, podía convertirse en el archivo a nivel nacional? ¿Para qué duplicar oficinas, con el consiguiente gasto y esfuerzo administrativo que esto generaría, si se podían subordinar las oficinas de las policías locales a la de la Capital y solucionar de esta manera el problema? El proyecto de ley proponía la creación de una oficina nacional dependiente del Ministerio de Justicia e Instrucción Pública, pero los argumentos sostenidos por los miembros de la comisión para independizar esta oficina de la policía no fueron tan contun-

${ }^{34}$ Diario de Sesiones del Cámara de Diputados de la Nación, Sesión del 12 de julio de 1905. Salvo indicación contraria, las citas siguientes corresponden a esta sesión. 
dentes como los de Quesada, sino que se centraron en la necesidad de darle un carácter nacional (y la policía de la Capital, aunque en muchos aspectos cumplía «de hecho» funciones federales, era una policía local) e internacional. Si bien no es explícito en el proyecto, se percibe una fuerte influencia de Vucetich, quien desde 1901 proponía la creación de oficinas de identificación internacionales que intercambiaran antecedentes (una en Europa, otra en Estados Unidos y la tercera en Argentina). La objeción fue desechada y el artículo se aprobó sin modificaciones.

Una segunda cuestión que generó un fuerte debate estuvo vinculada con el carácter estigmatizante de la identificación. El proyecto planteaba dos puntos polémicos: todos los procesados debían ser identificados cuando se les dictara prisión preventiva (esto, evidentemente, incluía a quienes serían absueltos o sobreseídos) y el archivo se formaría con los antecedentes de los procesados y condenados en todo el territorio de la República. Las posturas más extremas planteaban que los procesados no podían ser identificados. Claro que todo el proyecto perdía sentido si no se podía comprobar la reincidencia antes de la condena y no después. Los argumentos se basaban en lo habituales que resultaban las «órdenes de prisión preventiva, muchas veces con los informes falsos o intencionalmente confeccionados por la policía para hacer aparecer delincuente a una persona». Lo que resultaba afrentoso era, en primer lugar, el registro en un mismo archivo de honestos y delincuentes: «se trata de algo más que va a quedar allí como constancia de la oficina, que será un recuerdo triste para una persona que ha tenido la desgracia de ser sumariada", "criminales e inocentes quedarán perfectamente equiparados ante el sistema dactiloscópico». En los mismos casilleros se encontrarían mezcladas las fichas de procesados y condenados y quien hubiera resultado absuelto o sobreseído no se libraría del todo del proceso, porque sabría que sus huellas descansaban en el archivo, prolongando la «humillación» que había significado la detención preventiva. En segundo lugar, algunos diputados consideraban afrentosos los métodos de identificación biométrica: «una cosa es la identificación, y otra cosa es el escrutar el cuerpo de un procesado, para descubrirlo en todos los secretos y todos los detalles que pueda tener», la forma de identificación propuesta pretendía avanzar «hasta las partes más secretas del cuerpo».

¿Hasta qué punto el Estado podía extraer información del cuerpo, información que, en el caso de la huella digital, era ignorada incluso por el propio sujeto y sólo podía ser leída por expertos? ¿Por qué un hombre -o una mujer- inocente debía ser desnudado, medido y toda la superficie de su piel observada en busca de marcas 
y tatuajes? El proyecto sumaba a la dactiloscopia la antropometría y el sistema de filiación «provincia de Buenos Aires». Se trataba de una batería de métodos a los que se sometería al procesado: la huella digital revelaba una verdad acerca del sujeto sobre la que éste no tenía conocimiento, el bertillonage implicaba la medición y manipulación de todo el cuerpo y el sistema de filiación la observación minuciosa de su superficie para el registro de particularidades. Luego de arduas discusiones se aprobó la identificación de procesados pero con la destrucción de todos los registros de quienes fueran sobreseídos o absueltos y la conservación únicamente de los datos de identidad de quienes resultaran condenados.

En tercer lugar, se discutió si debía especificarse en la ley qué tipo de tecnologías tenían que utilizarse para la identificación y la legitimidad de la dactiloscopia para constituirse en prueba «concluyente y completa». El Artículo $4^{\circ}$ del proyecto planteaba que los «datos que para los fines de identificación, recoja o requiera esta oficina, se subordinarán a la clasificación dactiloscópica», a lo que se agregaba el sistema de filiación "provincia de Buenos Aires» y en algunos casos el bertillonage. $\mathrm{El}$ artículo $5^{\circ}$, más controvertido, planteaba que:

«La impresión digital será considerada como la prueba más concluyente y positiva de la identidad de un individuo y prevalecerá sobre las demás observaciones que sirvan para corroborarla».

Se discutió si era conveniente especificar en el cuerpo de la ley qué sistemas se utilizarían tanto para la identificación como para la clasificación de las fichas y se decidió quitar el artículo. Se trataba de una cuestión científica que podía cambiar con el tiempo: en algún momento se había sostenido que la antropometría era un sistema infalible y hoy se sabía que no era así, lo mismo podía suceder con la dactiloscopia en un futuro. También se cuestionó cuál era la calidad de prueba que debía otorgarse a la huella digital. En el proyecto se establecía que era una prueba concluyente, es decir, que el juez no podía evaluar otros elementos a la hora de determinar la identidad de una persona. Pero lo que hacía tan adecuada la dactiloscopia para su adopción en las instituciones policiales (la necesidad de pocos empleados sumamente calificados para la interpretación y clasificación de las huellas y la extraordinaria sencillez del proceso de toma, al revés de lo que sucedía con la antropometría), la volvía muy controvertida para jueces y legisladores. Mariano de Vedia diría que no le parecía 
«conveniente incorporar a las leyes declaraciones científicas, en cuyo caso, por lo menos, votaríamos la mayoría con la más absoluta ignorancia».

Como contracara de todas las ventajas que ofrecía la dactiloscopia sobre la antropometría y la fotografía, las desventajas para ser aceptada por los jueces eran grandes: al ojo del lego resultaban absolutamente indescifrables. Si bien la interpretación de la información antropométrica también necesitaba de cierto grado de expertise, cualquier persona con cierta formación podía entender al menos sus fundamentos. La dactiloscopia, en cambio, era verdaderamente contra intuitiva: en Argentina nunca antes la identidad humana había sido asociada a los dibujos de las yemas de los dedos; no existía ninguna similitud entre las impresiones digitales y otros rasgos visibles del sujeto (es más, nada decían sobre raza, sexo o edad); la afirmación científica según la cual no existían dos sujetos con las mismas impresiones digitales, ni que éstas podían modificarse con el paso del tiempo o por otros medios intencionales o naturales, era casi una profesión de fe. En estas condiciones, la nueva ciencia y sus expertos (todavía muy poco legitimados), ganaban en este proyecto un enorme poder en el proceso judicial.

Finalmente, recurriendo al argumento de que este tipo de cuestiones debían ser establecidas por los códigos locales de procedimientos en materia penal, ninguno de los dos artículos fue aceptado. El proyecto Gouchon no fue aprobado en la Cámara de Senadores y por muchos años se diluyó el intento de unificar la identificación criminal en todo el territorio.

\section{CONSIDERACIONES FINALES}

En 1933 se promulgó la primera ley de Registro nacional de reincidencia y estadística criminal y carcelaria ${ }^{35}$. Muchas de las propuestas de los proyectos de Quesada y Gouchon estaban presentes en esta ley. En primer lugar, el archivo se creó bajo la dependencia del Ministerio de Justicia e Instrucción Pública. En segundo lugar, la ley se consideró complementaria del Código Penal. En tercer lugar, los

procesados eran sistemáticamente identificados pero sus registros eran destruidos en caso de sobreseimiento o absolución. En cuarto lugar, los datos del registro fueron considerados prueba concluyente para comprobar la reincidencia (salvo que se demostrara error o falsedad). Por último, el objetivo del registro era comprobar

${ }^{35}$ Ley 11.752, Boletín Oficial, 13/10/1933. 
la reincidencia y brindar información a las policías para sus investigaciones, y sólo podía proporcionar informaciones bajo solicitud de juez o juzgado competente.

Habían pasado casi cuarenta años desde la sanción del Código Penal de 1886, años en los que el problema de la «delincuencia habitual» había sido fuertemente discutido y claramente visualizado como una amenaza al «orden social». Sin embargo, una serie de razones hicieron compleja la sistematización a escala nacional de la comprobación de la reincidencia.

El rechazo o la indiferencia ante las nuevas tecnologías de identificación en la justicia no sólo se pueden explicar por cuestiones culturales. La falta de exhaustividad de los archivos policiales, los frecuentes errores en las mediciones antropométricas y las filiaciones, la dificultad para aceptar como válido un nuevo tipo de evidencia que muy pocos podían evaluar, la no obligatoriedad de recurrir a las oficinas especializadas hizo que la identificación avanzara azarosamente en los tribunales. Por otro lado, el carácter local de los códigos de procedimientos en los que se especificaban las formas de identificar a los procesados dificultaba la creación de un archivo nacional, que necesariamente debía basar su clasificación en un único sistema. La construcción de este archivo demandaba la homogeneización de procedimientos y la construcción de una burocracia capaz de llevar adelante esta empresa identificatoria en todo el territorio.

Sin duda, la ofensa al honor que significaba estar registrado en archivos judiciales o policiales y la discusión sobre la legitimidad de la intervención estatal sobre el cuerpo de los ciudadanos fue un factor muy importante para detener los proyectos de ley en discusión y seguramente también para que jueces particulares decidieran no recurrir a las oficinas de identificación, pero estos factores deben ser puestos en relación con otras dificultades, complejizando la mirada sobre las relaciones entre identificación y justicia.

\section{Registro bibliográfico}

GARCÍA FERRARI, MERCEDES

«Una aproximación a las relaciones entre identificación y justicia en Argentina (1886-1933)», en: ESTUDIOS SOCIALES, revista universitaria semestral, año XXV, $n^{\circ} 48$, Santa Fe, Argentina, Universidad Nacional del Litoral, primer semestre de 2015, pp. 39-58.
Recibido: 18 / 12 / 2014

Aprobado: 15 / 02 / 2015 